\title{
The estimation and profile of the critical value for a Schrödinger equation
}

Jing Zeng*

\section{"Correspondence:}

zengjing@fjnu.edu.cn

School of Mathematics and

Computer Sciences, Fujian Normal

University, Fuzhou, 350007,

P.R. China

\begin{abstract}
In this paper, we are concerned with the following Schrödinger problem: $-\Delta u+V(x) u=f(u), u>0$, in $\mathbb{R}^{N}$, where $f: \mathbb{R} \rightarrow \mathbb{R}$ is of class $C^{1}$. The estimation and profile of the critical value of the corresponding functional is proved, which entails the relationship between the critical value on the balls and the least-energy value on the whole space. Our results are also true for three cases of the potential function $V(x)$.
\end{abstract}

Keywords: estimation; critical value; Schrödinger equation; potential function; compactness and noncompactness condition

\section{Introduction}

The main subject of this paper is the following problem:

$$
-\Delta u+V(x) u=f(u), \quad u>0, \text { in } \mathbb{R}^{N},
$$

where $\Delta=\sum_{i=1}^{N} \partial^{2} / \partial x_{i}^{2}$ is the Laplace operator. Compactness and noncompactness assumptions posed on the potential function $V(x)$ are also discussed.

The nonlinear Schrödinger equation (1.1) serves as a model for various problems in physics. For the last 20 years, (1.1) has received considerable attention as its solutions seem both mathematically intriguing and scientifically useful. We would like to mention earlier results on the existence of entire solutions of Schrödinger type equations with or without potentials, which was studied in [1-3] (see references therein).

A more general form of nonlinearity, i.e.

$$
-\Delta u+V(x) u=f(x, u), \quad u \in H^{1}\left(\mathbb{R}^{N}\right),
$$

is also studied by many authors. Kryszewski-Szulkin [4] considered the existence of a nontrivial solution of (1.2) in a situation when $f(x, u), V(x)$ are periodic in the $x$-variables, $f(x, u)$ is superlinear at $u=0$ and $|u|=\infty$, and 0 lies in a spectral gap of $-\Delta+V$. In addition, if $f(x, u)$ is odd in $u$, they proved that (1.2) has infinitely many solutions. The result from Bartsch-Wang [5] suggested that (1.2) should have one sign changing solution. Bartsch-Liu-Weth [6] further proved the existence of sign changing solutions of (1.2) in $H^{1}(\mathbb{R})$ with superlinear and subcritical nonlinearity $f(x, u)$, and the number of nodal domains can be controlled. If $f(x, u)$ is odd, they obtained an unbounded sequence of sign changing solutions $u_{k}(k \geq 1)$, and they have at most $k+1$ nodal domains.

(c) 2014 Zeng; licensee Springer. This is an Open Access article distributed under the terms of the Creative Commons Attribution License (http://creativecommons.org/licenses/by/4.0), which permits unrestricted use, distribution, and reproduction in any medium, provided the original work is properly credited. 
For the power nonlinearity $f(t)=t^{p}\left(1<p<\frac{N+2}{N-2}\right)$, since $V(x)>0$, it is well known that this problem has a positive solution which goes to zero at infinity. This solution is, besides, radially symmetric around some point and unique up to translations; see [7] and [8]. Moreover, the linearized equation around $w$ is nondegenerate in the sense that the equation

$$
\Delta u-V(x) u+p w^{p-1} u=0, \quad \text { in } \mathbb{R}^{N},
$$

has linear combinations of the functions $\partial w / \partial x_{i}$ as its only solutions which go to zero at infinity [8,9]. These facts are crucial in the formulation of a Lyapunov-Schmidt type procedure, which was first introduced by Floer-Weinstein [10] for the one-dimensional case, and then was extended by Oh [11,12] to higher dimensions. Ni-Wei [13] studied the critical value of the energy functional

$$
E(u)=\frac{1}{2} \int_{\Omega}\left(\varepsilon^{2}|\nabla u|^{2}+u^{2}\right)-\frac{1}{p+1} \int_{\Omega} u^{p+1}, \quad u \in H^{1}(\Omega),
$$

of the classical singular perturbation equation $-\varepsilon^{2} \Delta u+u=u^{p}(u>0)$ on $\Omega$, where $\Omega$ is a bounded smooth domain in $\mathbb{R}^{N}$.

Throughout this paper the following hypotheses on $f \in C^{1}$ and $V(x)$ will be assumed.

$\left(f_{1}\right) \quad f(0)=0, f(t)=o(|t|)$, as $|t| \rightarrow 0$, uniformly in $x$.

$\left(f_{2}\right)$ There exists a number $p>1$, with $p<\frac{N+2}{N-2}$ if $N \geq 3$, such that $\lim _{|t| \rightarrow \infty} \frac{F(t)}{|t|^{p}}<+\infty$, where $F(t)=\int_{0}^{t} f(s) d s$.

$\left(f_{3}\right) \quad$ There exists a number $\mu>2$ such that $0<\mu F(t) \leq t f(t)$, for all $|t|>0$.

$\left(f_{4}\right)$ For any $x, \frac{f^{\prime}(t) t-t}{f(t)-t}$ is nonincreasing in $t$.

$\left(V_{1}\right) \quad V \in C\left(\mathbb{R}^{N}, \mathbb{R}\right)$ and $\inf _{\mathbb{R}^{N}} V(x)>0$.

$\left(V_{2}\right) \quad V(x)=V(|x|)$.

Theorem 1.1 Under assumptions $\left(f_{1}\right) \sim\left(f_{3}\right)$ and $\left(V_{1}\right)$, there exists a least-energy ground state solution of (1.1).

Theorem 1.2 Under assumptions $\left(f_{1}\right) \sim\left(f_{4}\right)$ and $\left(V_{2}\right)$, there exists a least-energy ground state of (1.1), which is radially uniqueness solution, with the corresponding least-energy value $c_{*}$.

Remark 1.3 The assumptions $\left(V_{1}\right)$ and $\left(V_{2}\right)$ are adopted in [14], which means that the potential functions possess certain compactness conditions. $\left(V_{2}\right)$ indicates that the $x$ dependence is radially symmetric. For this case, $\left\{u \in H^{1}\left(\mathbb{R}^{N}\right) \mid \int_{\mathbb{R}^{N}} V(x) u^{2}<\infty, u\right.$ is radial $\}$ is compactly embedded in $L^{p}\left(\mathbb{R}^{N}\right)\left(2 \leq p<2^{*}=2 N /(N-2)\right)$.

Remark 1.4 By standard variational arguments, the assumptions $\left(f_{1}\right) \sim\left(f_{3}\right)$ and $\left(V_{1}\right)$ guarantee the results of Theorem 1.1, which can be proved by the traditional Minmax Theory. In fact, the critical value of the energy functional

$$
I(u)=\frac{1}{2} \int_{\mathbb{R}^{N}}\left(|\nabla u|^{2}+V(x) u^{2}\right)-\int_{\mathbb{R}^{N}} F(u), \quad u \in H^{1}\left(\mathbb{R}^{N}\right)
$$


can be characterized as

$$
c_{*}=\inf _{u \neq 0} \sup _{t>0} I(t u) .
$$

The associated critical point actually solves (1.1) and is called a least-energy solution. It decays exponentially at infinity.

Remark 1.5 As far as we know, the most general result of uniqueness of (1.1) type is obtained by Serrin-Tang [15], which would guarantee radial uniqueness in (1.1) if additionally one assumes $\left(f_{4}\right)$. The proof of Theorem 1.2 is similar to the steps in [15]; we omit the details.

Now we define the energy functional of (1.1) on $B_{\rho}=\left\{x \in \mathbb{R}^{N}|| x \mid \leq \rho\right\}$ :

$$
I_{\rho}(u)=\frac{1}{2} \int_{B_{\rho}}\left(|\nabla u|^{2}+V(x) u^{2}\right)-\int_{B_{\rho}} F(u) .
$$

The main result of this paper is the following.

Theorem 1.6 Under assumptions $\left(f_{1}\right) \sim\left(f_{4}\right)$ and $\left(V_{2}\right)$, the critical value $c_{\rho}$ of the functional $I_{\rho}$ satisfies

$$
c_{\rho}=c_{*}+\gamma^{2} \exp (-2 \rho(1+o(1))) \text {, }
$$

where $c_{*}$ is the least-energy value in Theorem 1.2 or Remark $1.4, \gamma$ is defined as

$$
\gamma=\frac{1}{2} \int_{\mathbb{R}^{N}} f(w) V>0,
$$

where $w$ is the unique solution in Theorem 1.2.

Remark 1.7 The assumptions $\left(f_{1}\right) \sim\left(f_{4}\right)$ and $\left(V_{2}\right)$ can guarantee the existence of $c_{\rho}$ on the bounded domain $B_{\rho}$. In fact, it can be proved by the Minmax Theory as in Remark 1.4.

Remark 1.8 Assuming the conditions $\left(V_{3}\right)$ or $\left(V_{4}\right)$ on $V(x)$, we can get a similar equality as (1.5).

$\left(V_{3}\right)$ There exists $r_{0}>0$ such that, for any $M>0$,

$$
\lim _{|y| \rightarrow \infty} m\left(\left\{x \in \mathbb{R}^{N}:|x-y| \leq r_{0}\right\} \cap\left\{x \in \mathbb{R}^{N}: V(x) \leq M\right\}\right)=0
$$

where $m$ denotes the Lebesgue measure on $\mathbb{R}^{N}$.

$\left(V_{4}\right) \quad V \in C\left(\mathbb{R}^{N}, \mathbb{R}\right), \inf _{\mathbb{R}^{N}} V(x)>0$, and $V(x) \rightarrow \infty$ as $|x| \rightarrow \infty$.

The assumptions $\left(V_{3}\right)$ and $\left(V_{4}\right)$ are certain compactness conditions, listed in [14]. $\left(V_{3}\right)$ is a more general condition, which gives a compact embedding. For $\left(V_{4}\right)$, we have a compact embedding from $\left\{u \in H^{1}\left(\mathbb{R}^{N}\right) \mid \int_{\mathbb{R}^{N}} V(x) u^{2}<\infty\right\}$ in $L^{p}\left(\mathbb{R}^{N}\right)$ for $2 \leq p<2^{*}$.

Remark 1.9 There are two cases of noncompactness conditions that are posed on the potential functions $V(x)$ [16], and the assumption $\left(V_{6}\right)$ is also adopted in [17]: 
$\left(V_{5}\right) V \in C\left(\mathbb{R}^{N}, \mathbb{R}\right), \inf _{\mathbb{R}^{N}} V(x) \geq V_{0}>0 . V(x)$ is 1-periodic in each of $x_{1}, x_{2}, \ldots, x_{N}$.

$\left(V_{6}\right) \quad 0<\inf _{\mathbb{R}^{N}} V(x) \leq \lim _{x \rightarrow \infty} V(x)=\sup _{\mathbb{R}^{N}} V(x)<\infty$.

Assumption $\left(V_{5}\right)$ is periodic, i.e., the $x$-dependence is periodic. $\left(V_{6}\right)$ means that $V(x)$ has a bounded potential well in the sense that $\lim _{|x| \rightarrow \infty} V(x)$ exists and is equal to $\sup _{\mathbb{R}^{N}} V(x)$.

Remark 1.10 A particularly interesting case is whether one can come to the same conclusion as Theorem 1.6 under the noncompact assumptions $\left(V_{5}\right)$ and $\left(V_{6}\right)$.

Our theorems generalize the results in [18] to three cases of compactness potential function entailing a type of nonlinear Schrödinger equation. The existence of the least-energy ground state solution of (1.1) is essential. Our results show the relationship between the critical value on the balls and the least-energy value on the whole space. The estimation of the critical value can be used to locate the geometrical shape of the solution.

\section{Preliminaries}

In this section, we give some preliminary lemmas, which will be adopted in the proof of the theorems.

Lemma 2.1 Assume $w$ is a solution of (1.1), and $w_{\rho}$ is a solution of

$$
\left\{\begin{array}{l}
-\Delta u+V(x) u=f(u), \quad u>0, \text { in } B_{\rho}, \\
u=0, \quad \text { on } \partial B_{\rho} .
\end{array}\right.
$$

Then

$$
\begin{aligned}
& w_{\rho}(\rho-1)=\gamma \exp (-\rho(1+o(1))), \\
& w(\rho-1)=\gamma \exp (-\rho(1+o(1))),
\end{aligned}
$$

where $\gamma$ is defined in (1.6).

Proof The proof of the two equalities is similar, we only prove the latter. Let $w$ be the unique positive solution of (1.1), then the function

$$
\bar{w}=w\left(\frac{x}{\sqrt{V(x)}}\right) V(x)^{\frac{\ln u}{\ln (u l f(u))}}
$$

satisfies the equation

$$
-\Delta u+u=f(u), \quad u>0, \text { in } \mathbb{R}^{N} .
$$

Next we consider the solution of the equation

$$
\left\{\begin{array}{l}
u^{\prime \prime}-\left(1-\frac{\tau}{2}\right) u=0, \quad \text { in }(R, \rho), \\
u(R)=1, \quad u(\rho)=0,
\end{array}\right.
$$

where $\rho>R$. 
Note $s=\sqrt{1-\frac{\tau}{2}}, h=e^{s R}-e^{2 s \rho-s R}$; then the solution of (2.3) is

$$
\bar{u}(x)=\frac{-e^{2 s \rho}}{h} e^{-s x}+\frac{1}{h} e^{s x} .
$$

We have $s \rightarrow 1$, as $\tau \rightarrow 0$. For $\rho$ big enough,

$$
\frac{1}{h}\left(e^{s \rho-s}-e^{s \rho+s}\right) \leq e^{-(1-\tau) \rho},
$$

So

$$
-\frac{e^{2 s \rho}}{h} e^{-s(\rho-1)}+\frac{1}{h} e^{s(\rho-1)} \leq e^{-(1-\tau) \rho}
$$

that is,

$$
\bar{u}(\rho-1) \leq e^{-(1-\tau) \rho} .
$$

The $\bar{u}$ in (2.4) is the supersolution of (2.2) on $[R, \rho)$. So on $[R, \rho), \bar{w} \leq \bar{u}$, we deduce that

$$
w\left(\frac{x}{\sqrt{V(x)}}\right) V(x)^{\frac{\ln u}{\ln (u / f(u))}} \leq e^{-(1-\tau) \rho},
$$

SO

$$
\lim _{r \rightarrow \infty} w(r) e^{(1-\tau) r}=\gamma>0,
$$

where $\gamma$ is defined in (1.6); then $w(\rho-1) \leq \gamma e^{-(1-\tau) \rho}$.

For the lower boundary estimation, given $R>0$, consider the equation

$$
\left\{\begin{array}{l}
u^{\prime \prime}+\frac{N-1}{R} u^{\prime}-u=0, \quad \text { in }(R, \rho), \\
u(R)=w_{\rho}(R), \quad u(\rho)=0 .
\end{array}\right.
$$

Similarly to the computation of (2.3), we get, for $\rho$ big enough, $\underline{u}(\rho-1) \geq e^{-(1-\tau) \rho}$, and $\underline{u}$ is a subsolution of the above equation. So $\bar{w} \geq \underline{u}$. Therefore, for $\rho$ big enough, $w\left(\frac{x}{\sqrt{V(x)}}\right) V(x)^{\frac{\ln u}{\ln (u / f(u))}} \geq e^{-(1-\tau) \rho}$, so $w(\rho-1) \geq \gamma e^{-\rho(1+o(1))}$. We conclude that $w(\rho-1)=$ $\gamma e^{-\rho(1+o(1))}$.

Lemma 2.2 Let u be a solution of

$$
\left\{\begin{array}{l}
u^{\prime \prime}-u=0, \quad \text { in }(\rho-1, \infty) \\
u(\rho-1)=1, \quad u(+\infty)=0 .
\end{array}\right.
$$

Let $v$ be a solution of

$$
\begin{cases}v^{\prime \prime}-h(\rho) v=0, & \text { in }(\rho-1, \rho), \\ v(\rho-1)=1, & v(\rho)=0,\end{cases}
$$

where $h(\rho)$ is a function of $\rho$. Then $v^{\prime}(\rho-1)-u^{\prime}(\rho-1) \geq 1$. 
Proof By computation $u=e^{\rho-1} \cdot e^{-x}$ is a solution of $(2.5)$, and $u^{\prime}(\rho-1)=-1$. Similarly, it can be checked that

$$
\nu=\frac{-e^{\sqrt{h(\rho)} \cdot \rho}}{e^{-\sqrt{h(\rho)}}-e^{\sqrt{h(\rho)}}} \cdot e^{-\sqrt{h(\rho)} \cdot x}+\frac{1}{e^{\sqrt{h(\rho)} \cdot \rho}\left(e^{-\sqrt{h(\rho)}}-e^{\sqrt{h(\rho)}}\right)} \cdot e^{\sqrt{h(\rho) \cdot x}}
$$

is a solution of (2.6), and

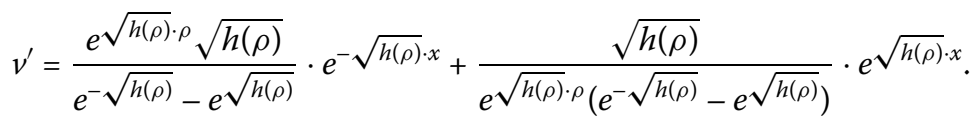

So $v^{\prime}(\rho-1) \geq 0=u^{\prime}(\rho-1)+1$, i.e. $v^{\prime}(\rho-1)-u^{\prime}(\rho-1) \geq 1$.

\section{The estimation of the critical value}

This section is devoted to the proof of Theorem 1.6.

Proof of Theorem $1.6 c_{\rho}$ is the critical value of the functional $I_{\rho}, c_{*}$ is the least-energy value of $I(u)$.

First we find the upper bound of $c_{\rho}$. Let $v_{\rho}$ be the solution of the equation

$$
\left\{\begin{array}{lr}
-\Delta u+V(x) u=0, & \text { in } B_{\rho} \backslash B_{\rho-1} \\
u(\rho-1)=w(\rho-1), & u(\rho)=0
\end{array}\right.
$$

where $w$ is the solution of (1.1) in Theorem 1.2, then

$$
\int_{B_{\rho} \backslash B_{\rho-1}} V(x) v_{\rho}^{2}=\int_{B_{\rho} \backslash B_{\rho-1}} \Delta v_{\rho} \cdot v_{\rho}=\left.\nabla v_{\rho} \cdot v_{\rho}\right|_{\partial B_{\rho-1}}-\int_{B_{\rho} \backslash B_{\rho-1}}\left|\nabla v_{\rho}\right|^{2},
$$

so

$$
\int_{B_{\rho} \backslash B_{\rho-1}}\left|\nabla v_{\rho}\right|^{2}+\int_{B_{\rho} \backslash B_{\rho-1}} V(x) v_{\rho}^{2}=\left.\nabla v_{\rho} \cdot v_{\rho}\right|_{\partial B_{\rho-1}} .
$$

Define

$$
\bar{w}_{\rho}(r)= \begin{cases}w(r), & 0 \leq r \leq \rho-1 \\ v_{\rho}(r), & \rho-1 \leq r \leq \rho\end{cases}
$$

We have

$$
c_{\rho}=I_{\rho}\left(w_{\rho}\right)=\max _{t \geq 0} I_{\rho}\left(t w_{\rho}\right) \leq \max _{t \geq 0} I_{\rho}\left(t \bar{w}_{\rho}\right)=I_{\rho}\left(t_{\rho} \bar{w}_{\rho}\right),
$$

and

$$
t_{\rho} \rightarrow 1, \quad \text { as } \rho \rightarrow \infty .
$$


Moreover, by the definition of $\bar{w}_{\rho}$,

$$
\begin{aligned}
I_{\rho}\left(t_{\rho} \bar{w}_{\rho}\right)= & \frac{1}{2} \int_{B_{\rho}} t_{\rho}^{2}\left(\left|\nabla \bar{w}_{\rho}\right|^{2}+V(x) \bar{w}_{\rho}^{2}\right)-\int_{B_{\rho}} F\left(t_{\rho} \bar{w}_{\rho}\right) \\
= & \frac{1}{2} \int_{B_{\rho-1}} t_{\rho}^{2}\left(\left|\nabla \bar{w}_{\rho}\right|^{2}+V(x) \bar{w}_{\rho}^{2}\right)-\int_{B_{\rho-1}} F\left(t_{\rho} \bar{w}_{\rho}\right) \\
& +\left(\frac{1}{2} \int_{B_{\rho} \backslash B_{\rho-1}} t_{\rho}^{2}\left(\left|\nabla \bar{w}_{\rho}\right|^{2}+V(x) \bar{w}_{\rho}^{2}\right)-\int_{B_{\rho} \backslash B_{\rho-1}} F\left(t_{\rho} \bar{w}_{\rho}\right)\right) \\
\leq & \frac{1}{2} \int_{B_{\rho-1}} t_{\rho}^{2}\left(|\nabla w|^{2}+V(x) w^{2}\right)-\int_{B_{\rho-1}} F\left(t_{\rho} w\right) \\
& +\frac{1}{2} \int_{B_{\rho} \backslash B_{\rho-1}} t_{\rho}^{2}\left(\left|\nabla v_{\rho}\right|^{2}+V(x) v_{\rho}^{2}\right) \\
\leq & c_{*}+\left.t_{\rho}^{2} \cdot \nabla v_{\rho} \cdot v_{\rho}\right|_{\partial B_{\rho-1}} \\
\leq & c_{*}+\left.t_{\rho}^{2} \cdot v_{\rho}^{\prime}(r) \cdot v_{\rho}(r)\right|_{r=\rho-1},
\end{aligned}
$$

where (3.1) is used in the second inequality.

Similar to the computation in Lemma 2.1, we have

$$
\begin{aligned}
& v_{\rho}^{\prime}(\rho-1) \leq \gamma \exp (-\rho(1+o(1))) \\
& v_{\rho}(\rho-1) \leq \gamma \exp (-\rho(1+o(1))) .
\end{aligned}
$$

Combine (3.2), (3.3), (3.4), (3.5), and (3.6), then

$$
c_{\rho} \leq c_{*}+\gamma^{2} \exp (-2 \rho(1+o(1))) \text {. }
$$

Next we find the lower bound of $c_{\rho}$. Let $\bar{v}_{\rho}$ be the solution of

$$
\left\{\begin{array}{l}
-\Delta u+V(r) u=0, \quad \text { in } \mathbb{R}^{N} \backslash B_{\rho-1}, \\
u(\rho-1)=w_{\rho}(\rho-1), \quad u(+\infty)=0
\end{array}\right.
$$

then

$$
\int_{\mathbb{R}^{N} \backslash B_{\rho-1}} V \bar{v}_{\rho}^{2}=\int_{\mathbb{R}^{N} \backslash B_{\rho-1}} \Delta \bar{v}_{\rho} \cdot \bar{v}_{\rho}=\left.\nabla \bar{v}_{\rho} \cdot \bar{v}_{\rho}\right|_{\partial B_{\rho-1}}-\int_{\mathbb{R}^{N} \backslash B_{\rho-1}}\left|\nabla \bar{v}_{\rho}\right|^{2},
$$

so

$$
\int_{\mathbb{R}^{N} \backslash B_{\rho-1}}\left|\nabla \bar{v}_{\rho}\right|^{2}+V \bar{v}_{\rho}^{2}=\left.\nabla \bar{v}_{\rho} \cdot \bar{v}_{\rho}\right|_{\partial B_{\rho-1}} \cdot
$$

Define

$$
\hat{w}_{\rho}(r)= \begin{cases}w_{\rho}(r), & 0 \leq r \leq \rho-1 \\ \bar{v}_{\rho}(r), & \rho-1 \leq r \leq \infty\end{cases}
$$


Zens Boundary Value Problems 2014, 2014:202

Page 8 of 10

For $t \geq 0$,

$$
\begin{aligned}
I_{\rho}\left(w_{\rho}\right)= & \frac{1}{2} \int_{B_{\rho}}\left(\left|\nabla w_{\rho}\right|^{2}+V w_{\rho}^{2}\right)-\int_{B_{\rho}} F\left(w_{\rho}\right) \\
= & \left(\frac{1}{2} \int_{B_{\rho-1}}\left(\left|\nabla w_{\rho}\right|^{2}+V w_{\rho}^{2}\right)-\int_{B_{\rho-1}} F\left(w_{\rho}\right)\right) \\
& +\left(\frac{1}{2} \int_{B_{\rho} \backslash B_{\rho-1}}\left(\left|\nabla w_{\rho}\right|^{2}+V w_{\rho}^{2}\right)-\int_{B_{\rho} \backslash B_{\rho-1}} F\left(w_{\rho}\right)\right) .
\end{aligned}
$$

For the second part in (3.8), by $I_{\rho}\left(w_{\rho}\right)=\max _{t>0} I_{\rho}\left(t w_{\rho}\right)$, for $t>0$,

$$
\begin{aligned}
& \frac{1}{2} \int_{B_{\rho} \backslash B_{\rho-1}}\left(\left|\nabla w_{\rho}\right|^{2}+V w_{\rho}^{2}\right)-\int_{B_{\rho} \backslash B_{\rho-1}} F\left(w_{\rho}\right) \\
& \quad \geq \frac{t^{2}}{2} \int_{B_{\rho} \backslash B_{\rho-1}}\left(\left|\nabla w_{\rho}\right|^{2}+V w_{\rho}^{2}\right)-\int_{B_{\rho} \backslash B_{\rho-1}} F\left(t w_{\rho}\right) \\
& \quad \geq \frac{1}{2} \int_{B_{\rho} \backslash B_{\rho-1}}\left(t^{2}\left|\nabla w_{\rho}\right|^{2}+\max _{\rho-1 \leq r \leq \rho}\left\{V-\frac{F\left(t w_{\rho}\right)}{\left(t w_{\rho}\right)^{2}}\right\} t^{2} w_{\rho}^{2}\right) .
\end{aligned}
$$

So

$$
\begin{aligned}
I_{\rho}\left(t w_{\rho}\right)= & \frac{t^{2}}{2} \int_{B_{\rho}}\left(\left|\nabla w_{\rho}\right|^{2}+V w_{\rho}^{2}\right)-\int_{B_{\rho}} F\left(t w_{\rho}\right) \\
\geq & \frac{t^{2}}{2} \int_{B_{\rho-1}}\left(\left|\nabla w_{\rho}\right|^{2}+V w_{\rho}^{2}\right)-\int_{B_{\rho-1}} F\left(t w_{\rho}\right) \\
& +\frac{1}{2} \int_{B_{\rho} \backslash B_{\rho-1}}\left(t^{2}\left|\nabla w_{\rho}\right|^{2}+\max _{\rho-1 \leq r \leq \rho}\left\{V-\frac{F\left(t w_{\rho}\right)}{\left(t w_{\rho}\right)^{2}}\right\} t^{2} w_{\rho}^{2}\right) \\
= & \frac{t^{2}}{2} \int_{B_{\rho-1}}\left(\left|\nabla w_{\rho}\right|^{2}+V w_{\rho}^{2}\right)-\int_{B_{\rho-1}} F\left(t w_{\rho}\right) \\
& +\frac{t^{2}}{2} \int_{\mathbb{R}^{N} \backslash B_{\rho-1}}\left(\left|\nabla \bar{v}_{\rho}\right|^{2}+V \bar{v}_{\rho}^{2}\right)-\frac{t^{2}}{2} \int_{\mathbb{R}^{N} \backslash B_{\rho-1}}\left(\left|\nabla \bar{v}_{\rho}\right|^{2}+V \bar{v}_{\rho}^{2}\right) \\
& +\frac{1}{2} \int_{B_{\rho} \backslash B_{\rho-1}} t^{2}\left|\nabla w_{\rho}\right|^{2}+\frac{1}{2} \int_{B_{\rho} \backslash B_{\rho-1}} \max _{\rho-1 \leq r \leq \rho}\left\{V-\frac{F\left(t w_{\rho}\right)}{\left(t w_{\rho}\right)^{2}}\right\} t^{2} w_{\rho}^{2} \\
\geq & \frac{t^{2}}{2} \int_{B_{\rho-1}}\left(\left|\nabla w_{\rho}\right|^{2}+V w_{\rho}^{2}\right)-\int_{B_{\rho-1}} F\left(t w_{\rho}\right)+\frac{t^{2}}{2} \int_{\mathbb{R}^{N} \backslash B_{\rho-1}}\left(\left|\nabla \bar{v}_{\rho}\right|^{2}+V \bar{v}_{\rho}^{2}\right) \\
& -\int_{\mathbb{R}^{N} \backslash B_{\rho-1}} F\left(t \bar{v}_{\rho}\right)-\frac{t^{2}}{2} \int_{\mathbb{R}^{N} \backslash B_{\rho-1}}\left(\left|\nabla \bar{v}_{\rho}\right|^{2}+V \bar{v}_{\rho}^{2}\right) \\
& +\frac{1}{2} \int_{B_{\rho} \backslash B_{\rho-1}} t^{2}\left|\nabla w_{\rho}\right|^{2}+\frac{1}{2} \int_{B_{\rho} \backslash B_{\rho-1}} \max _{\rho-1 \leq r \leq \rho}\left\{V-\frac{F\left(t w_{\rho}\right)}{\left(t w_{\rho}\right)^{2}}\right\} t^{2} w_{\rho}^{2}, \\
= & I\left(t \hat{w}_{\rho}\right)-\frac{t^{2}}{2} \int_{\mathbb{R}^{N} \backslash B_{\rho-1}}\left(\left|\nabla \bar{v}_{\rho}\right|^{2}+V \bar{v}_{\rho}^{2}\right)+\frac{1}{2} \int_{B_{\rho} \backslash B_{\rho-1}} t^{2}\left|\nabla w_{\rho}\right|^{2} \\
& \left.\left.\max _{\rho}\right)^{2}\right\} t^{2} w_{\rho}^{2} \\
& \\
& \\
&
\end{aligned}
$$


where $I$ is defined in (1.3). Take (3.7) in the last equality of the above, then

$$
\begin{aligned}
I_{\rho}\left(t w_{\rho}\right) \geq & I\left(t \hat{w}_{\rho}\right)-\left.\frac{t^{2}}{2} \nabla \bar{v}_{\rho} \cdot \bar{v}_{\rho}\right|_{\partial B_{\rho-1}}+\frac{1}{2} \int_{B_{\rho} \backslash B_{\rho-1}} t^{2}\left|\nabla w_{\rho}\right|^{2} \\
& +\frac{1}{2} \int_{B_{\rho} \backslash B_{\rho-1}} \max _{\rho-1 \leq r \leq \rho}\left\{V-\frac{F\left(t w_{\rho}\right)}{\left(t w_{\rho}\right)^{2}}\right\} t^{2} w_{\rho}^{2} .
\end{aligned}
$$

Choose $t_{\rho}$ such that $I\left(t_{\rho} \hat{w}_{\rho}\right) \geq c_{*}$, and let $w_{\rho} \rightarrow w$ in $H^{1}$, then as $\rho \rightarrow \infty, t_{\rho} \rightarrow 1$. Moreover, $\max _{\rho-1 \leq r \leq \rho}\left\{V-\frac{F\left(t_{\rho} w_{\rho}\right)}{\left(t_{\rho} w_{\rho}\right)^{2}}\right\} \rightarrow \max _{\rho-1 \leq r \leq \rho} V$, as $\rho \rightarrow \infty$.

Next we consider $\hat{v}_{\rho}$, which gives the solution of

$$
\left\{\begin{array}{l}
-\Delta u+\max \left\{V-\frac{F\left(t_{\rho} w_{\rho}\right)}{\left(t_{\rho} w_{\rho}\right)^{2}}\right\} u=0, \quad \text { in } B_{\rho} \backslash B_{\rho-1}, \\
u(\rho-1)=w_{\rho}(\rho-1), \quad u(\rho)=0 .
\end{array}\right.
$$

Here $\hat{v}_{\rho}$ works as the comparison function. Similarly to the computation of (3.1) and (3.7),

$$
\begin{aligned}
& \int_{B_{\rho} \backslash B_{\rho-1}} \max _{\rho-1 \leq r \leq \rho}\left\{V-\frac{F\left(t_{\rho} w_{\rho}\right)}{\left(t_{\rho} w_{\rho}\right)^{2}}\right\} \hat{v}_{\rho}^{2} \\
& =\int_{B_{\rho} \backslash B_{\rho-1}} \Delta \hat{v}_{\rho} \cdot \hat{v}_{\rho} \\
& =\left.\nabla \hat{v}_{\rho} \cdot \hat{v}_{\rho}\right|_{\partial B_{\rho-1}}-\int_{B_{\rho} \backslash B_{\rho-1}}\left|\nabla \hat{v}_{\rho}\right|^{2},
\end{aligned}
$$

that is,

$$
\int_{B_{\rho} \backslash B_{\rho-1}}\left(\left|\nabla \hat{v}_{\rho}\right|^{2}+\max _{\rho-1 \leq r \leq \rho}\left\{V-\frac{F\left(t_{\rho} w_{\rho}\right)}{\left(t_{\rho} w_{\rho}\right)^{2}}\right\} \hat{v}_{\rho}^{2}\right)=\left.\nabla \hat{v}_{\rho} \cdot \hat{v}_{\rho}\right|_{\partial B_{\rho-1}} .
$$

Take (3.10) in (3.9); by the definition of $\bar{v}_{\rho}$ and $\hat{v}_{\rho}$, then

$$
\begin{aligned}
I_{\rho}\left(t w_{\rho}\right) \geq & I\left(t_{\rho} \hat{w}_{\rho}\right)-\left.\frac{t_{\rho}^{2}}{2} \nabla \bar{v}_{\rho} \cdot \bar{v}_{\rho}\right|_{\partial B_{\rho-1}}+\frac{t_{\rho}^{2}}{2} \int_{B_{\rho} \backslash B_{\rho-1}}\left|\nabla \hat{v}_{\rho}\right|^{2} \\
& +\frac{t_{\rho}^{2}}{2} \int_{B_{\rho} \backslash B_{\rho-1}} \max _{\rho-1 \leq r \leq \rho}\left\{V-\frac{F\left(t_{\rho} w_{\rho}\right)}{\left(t_{\rho} w_{\rho}\right)^{2}}\right\} \hat{v}_{\rho}^{2} \\
= & I\left(t_{\rho} \hat{w}_{\rho}\right)-\left.\frac{t_{\rho}^{2}}{2} \nabla \bar{v}_{\rho} \cdot \bar{v}_{\rho}\right|_{\partial B_{\rho-1}}+\left.\frac{t_{\rho}^{2}}{2} \nabla \hat{v}_{\rho} \cdot \hat{v}_{\rho}\right|_{\partial B_{\rho-1}} \\
\geq & c_{*}+\frac{t_{\rho}^{2}}{2} w_{\rho}(\rho-1)\left(\hat{v}_{\rho}^{\prime}(\rho-1)-\bar{v}_{\rho}^{\prime}(\rho-1)\right) .
\end{aligned}
$$

By Lemma 2.2, $\hat{v}_{\rho}^{\prime}(\rho-1)-\bar{v}_{\rho}^{\prime}(\rho-1) \geq w_{\rho}(\rho-1)$. And by Lemma 2.1 and (3.11),

$$
c_{\rho}=I_{\rho}\left(w_{\rho}\right)=\max _{t>0} I_{\rho}\left(t w_{\rho}\right) \geq c_{*}+\gamma^{2} \exp (-2 \rho(1+o(1))) .
$$

So we conclude

$$
c_{\rho}=c_{*}+\gamma^{2} \exp (-2 \rho(1+o(1))) \text {. }
$$




\section{Competing interests}

The author declares to have no competing interests.

\section{Author's contributions}

The work was carried out by the author.

\section{Acknowledgements}

The author is supported by China Postdoctoral Science Foundation (2014M551830) and the Nonlinear Analysis Innovation Team (IRTL1206) funded by Fujian Normal University.

\section{Received: 30 July 2014 Accepted: 14 August 2014 Published online: 25 September 2014}

\section{References}

1. Berestycki, H, Lions, PL: Nonlinear scalar field equations. I. Existence of a ground state. Arch. Ration. Mech. Anal. 82, 313-345 (1983)

2. Ding, WY, Ni, WM: On the existence of positive entire solutions of a semilinear elliptic equation. Arch. Ration. Mech. Anal. 91, 283-308 (1986)

3. Rabinowitz, PH: On a class of nonlinear Schrödinger equations. Z. Angew. Math. Phys. 43, 270-291 (1992)

4. Kryszewski, W, Szulkin, A: Generalized linking theorem with an application to semilinear Schrödinger equation. Adv. Differ. Equ. 3, 441-472 (1998)

5. Bartsch, T, Wang, ZQ: On the existence of sign changing solutions for semilinear Dirichlet problems. Topol. Methods Nonlinear Anal. 7, 115-131 (1999)

6. Bartsch, T, Liu, ZL, Weth, T: Sign changing solutions of superlinear Schrödinger equations. Commun. Partial Differ. Equ. 29, 25-42 (2004)

7. Gidas, B, Ni, WM, Nirenberg, L: Symmetry of positive solutions of nonlinear elliptic equations in $\mathbb{R}^{N}$. Adv. Math. Stud. A 7, 209-243 (1979)

8. Kwong, MK: Uniqueness of positive solutions of $\Delta u-u+u^{p}=0$ in $\mathbb{R}^{n}$. Arch. Ration. Mech. Anal. 105, 243-266 (1989)

9. Ni, WM, Takagi, I: On the shape on least-energy solutions to a semilinear Neumann problem. Commun. Pure Appl. Math. XLIV, 819-851 (1991)

10. Floer, A, Weinstein, A: Nonspreading wave packets for the cubic Schrödinger equation with a bounded potential. J. Funct. Anal. 69, 397-408 (1986)

11. Oh, YJ: Existence of semi-classical bound states of nonlinear Schrödinger equations with potential on the class $(V)_{a}$. Commun. Partial Differ. Equ. 13, 1499-1519 (1988)

12. Oh, YJ: On positive multi-lump bound states nonlinear Schrödinger equations under multiple well potential. Commun. Math. Phys. 131, 223-253 (1990)

13. Ni, WM, Wei, J: On the location and profile of spike-layer solutions to singularly perturbed semilinear Dirichlet problems. Commun. Pure Appl. Math. 48, 731-768 (1995)

14. Liu, Z, Wang, ZQ: On the Ambrosetti-Rabinowitz superlinear condition. Adv. Nonlinear Stud. 4, 561-572 (2004)

15. Serrin, J, Tang, M: Uniqueness of ground states for quasilinear elliptic equations. Indiana Univ. Math. J. 49, 897-923 (2000)

16. Li, YQ, Wang, ZQ, Zeng, J: Ground states of nonlinear Schrödinger equations with potentials. Ann. Inst. Henri Poincaré Anal. Non Linéaire 23, 829-837 (2006)

17. Jeanjean, L, Tanaka, K: A positive solution for a nonlinear Schrödinger equation on $\mathbb{R}^{N}$. Indiana Univ. Math. J. 54 443-464 (2005)

18. Del Pino, M, Felmer, PL: Spike-layered solutions of singularly perturbed elliptic problems in a degenerate setting. Indiana Univ. Math. J. 48, 883-898 (1999)

doi:10.1186/s13661-014-0202-7

Cite this article as: Zeng: The estimation and profile of the critical value for a Schrödinger equation. Boundary Value Problems 2014 2014:202.

\section{Submit your manuscript to a SpringerOpen ${ }^{\circ}$ journal and benefit from:}

- Convenient online submission

- Rigorous peer review

- Immediate publication on acceptance

- Open access: articles freely available online

- High visibility within the field

- Retaining the copyright to your article 